\title{
A Model for the Optimization of the Shale Gas Horizontal Well Section Based on the Combination of Different Weighting Methods in the Frame of the Game Theory
}

\author{
Minxuan $\mathrm{Li}^{1}$, Jing Sun ${ }^{1, *}$, Dehua Liu ${ }^{1}$, Yang $\mathrm{Li}^{1}$, Kuidong $\mathrm{Li}^{2}$, Wei Liu ${ }^{2}$, Jialin $\mathrm{Xiao}^{2}$ and Jiani $\mathrm{Hu}^{3}$ \\ ${ }^{1}$ School of Petroleum Engineering, Yangtze University, Wuhan, 430100, China \\ ${ }^{2}$ Sinopec Jianghan Oilfield Research Institute of Petroleum Engineering, Wuhan, 430035, China \\ ${ }^{3}$ PetroChina Qinghai Oilfield Company, Dunhuang, 736200, China \\ ${ }^{*}$ Corresponding Author: Jing Sun. Email: sunjing_email@163.com \\ Received: 05 March 2020; Accepted: 03 August 2020
}

\begin{abstract}
Existing "evaluation indicators" are selected and combined to build a model to support the optimization of shale gas horizontal wells. Towards this end, different "weighting methods", including AHP and the so-called entropy method, are combined in the frame of the game theory. Using a relevant test case for the implementation of the model, it is shown that the horizontal section of the considered well is in the middle sweet spot area with good physical properties and fracturing ability. In comparison with the FSI (flow scanner Image) gas production profile, the new model seems to display better abilities for the optimization of horizontal wells.
\end{abstract}

Keywords: Combination weight; well section sweet spot; AHP; entropy method; evaluation model

\section{Introduction}

Shale gas is an unconventional natural gas, found in its adsorbed or free state principally in black shale or high-carbon shale [1]. The shale gas resources in China are huge. After many years of exploration, the industrial exploitation of marine shale gas in the Sichuan Basin has begun to be realized [2]. However, when fracturing the target formation and preparing the horizontal shale gas well, it is often assumed that the shale reservoir is homogeneous. As a result, uniform fracturing processes are typically adopted. However, a large number of domestic gas production profiling studies have shown that horizontal well sections basically conform to a limited "three by three by three" principle [3].

The evaluation of how to position the fractured interval in a horizontal well basically involves determining a "sweet spot" at different levels. A sweet spot can be informed by engineering considerations and geological considerations.

Drawing upon the characteristics of China's marine shale reservoirs, the author has examined a large amount of data regarding block sweet spots, layer sweet spots, and the sweet spots in horizontal well sections [4-6]. There are often two problems. First of all, the selection of evaluation indicators can be redundant and unrepresentative, or key indicators may be missing. Secondly, the selection of indicator

This work is licensed under a Creative Commons Attribution 4.0 International License, which permits unrestricted use, distribution, and reproduction in any medium, provided the original work is properly cited. 
weights can be too subjective. For example, Wang et al. [7] used the four parameters of organic carbon content, vitrinite reflectance, shear modulus and fracture toughness as the key parameters for horizontal well section optimization. However, the key indicator of free gas-related porosity is not considered. Lai et al. [8] use subjective criteria to select the weight given to various parameters in their study, without allowing for any objective regularity. To sum up, the establishment of a set of reasonable evaluation indicators and an effective horizontal well section evaluation model is needed to improve the development of shale gas horizontal wells. In this paper, a sweet spot indicator for horizontal well sections and an improved system for evaluation will first be established. A combined weight method based on game theory is then used to calculate the weight of different horizontal well section evaluation indicators. Standardized horizontal well section evaluation indicator values and horizontal well section evaluation indicator weights are combined by means of multiplication synthesis and a refined evaluation model for horizontal well sections is then established. The target layer can be divided by incorporating the baseline indicator values of good, medium and poor into the evaluation model, making the evaluation results more directly available. Finally, well A1 in the Fuling District of Chongqing is used as an example to verify the proposed approach.

\section{Determination of Sweet Spot Indicators and the Evaluation System}

\subsection{Sweet Spot Indicator Determination and Screening}

Sweet spot indicators from both an engineering and geological perspective form a fundamental part the evaluation of shale gas horizontal well sections. According to prior research [9] and practical application, the main engineering sweet spot indicators can be summarized as follows: brittleness indicators; the minimum horizontal principal stress; brittle minerals (calcium, shale content); fracturing indicators; Young's modulus; Poisson's ratio; fracture pressure; the stress difference coefficient; shale content; toughness indicators; property indicators; fracture permeability; and the shear modulus. The principal geological sweet spot indicators are: organic carbon content; organic maturity; gas content; porosity; burial depth; formation pressure; and target layer thickness. To identify the most effective indicators, the various sweet spot indicators were screened.

Amongst the engineering sweet spot indicators, the minimum horizontal stress, brittle minerals, Young's modulus, Poisson's ratio, formation fracture pressure, stress difference coefficient, and shear modulus are all related to the brittleness indicator. Therefore, to limit the high correlation of some indicators caused by having too many with an overlapping focus, it seemed best to concentrate on engineering sweet spot indicators that are specifically needed to establish an evaluation model for horizontal well sections. As a result, the brittleness indicator, toughness indicator and fracturing indicator were retained as the main factors. With regard to the geological sweet spot indicators, the ones related to the target layer were retained, while the burial depth, formation pressure, layer thickness, and organic matter maturity are typically assigned fixed values, so they were not considered. Thus, the chosen geological sweet spot indicators were: the organic carbon content; permeability; porosity; and gas content. To sum up, the overall sweet spots indicators used to model horizontal well sections were: the brittleness indicator; toughness indicator; fracturing indicator; organic carbon content; permeability; porosity; and gas content.

\subsubsection{Brittleness Indicator}

The rock brittleness indicator is the ratio between its compressive strength and tensile strength. It is used to indicate how susceptible a rock is to cracking. Results here suggest that the brittleness indicator changes when artificial fractures take different forms. So, reconstruction of the reservoir accessed by fracturing is directly affected by the brittleness indicator. 
The most commonly-used calculation equation for the brittleness indicator is:

$B_{m}=\left(\frac{E_{t}-E_{t \text { min }}}{E_{t \max }-E_{t \min }}+\frac{\gamma_{t}-\gamma_{t \max }}{\gamma_{t \min }-\gamma_{t \max }}\right) / 2$.

where: $B_{m}$ is the brittleness indicator; $E_{t}$ is Young's modulus of elasticity relating to shale formations; $E_{t \max }$ and $E_{t \max }$ are the maximum and minimum value of Young's modulus, respectively; $\gamma_{t}$ is Poisson's ratio for shale formations; $\gamma_{t \max }$ and $\gamma_{t \text { min }}$ are the maximum and minimum values for Poisson's ratio, respectively. The mechanical parameters used in the above equation can be calculated from well completion data.

\subsubsection{Toughness Indicator}

The toughness indicator of rock is a product of type I and type II fracture toughness, i.e.:

$K=K_{I C} \bullet K_{I I C}$.

This equation expresses the effect of the toughness on reservoir compressibility. The critical value is the rock stress intensity factor. This determines the force needed in the fracturing process. Its particular physical importance is that attention to this is required to prevent over-extension of the fracture. The smaller the fracture toughness value, the easier it is for the fracture to be extended and the greater the rock's compressibility. Shale gas volume fracturing typically uses Type I (open) and Type II (staggered) fractures. Jin et al. [10] have conducted a number of practical experiments and have established the equations for calculating Type I and Type II fracture toughness:

$K_{I C}=0.2176 P_{w}+0.0059 S_{t}^{3}+0.0923 S_{t}^{3}+0.517 S_{t}-0.3322$.

$K_{I I C}=0.0956 p_{w}+0.1383 S_{t}-0.082$.

$K$ is the toughness indicator; $K_{I C}$ and $K_{I I C}$ are the type I and type II fracture toughness, respectively; $P_{w}$ is the confining pressure; and $S_{t}$ is the rock tensile strength. $p_{w}$ and $S_{t}$ can be calculated by combining well $\log$ data and empirical equations [11].

\subsubsection{Fracturing Indicator}

The fracturing indicator [12], which combines the brittleness indicator and toughness indicator, indicates the difficulty of fracturing the reservoir:

$F_{f r e}=\frac{2 B}{K_{I C} K_{I I C}}$.

$F_{f r e}$ is the brittleness indicator; and $\overline{K_{I C} K_{I I C}}$ represents the multiplied average values for the Type I and Type II fracture toughness.

\subsubsection{Total Organic Carbon Content (TOC)}

The TOC is an important indicator that evaluates the grade of the source rock. It changes with the quantity of adsorbed gas in the shale and ultimately indicates the gas production rate for a shale gas reservoir. The TOC is difficult to obtain experimentally. In this paper, we use well log and completion data as a reference [13] and perform a TOC regression calculation, according to the following:

TOC $=-0.16129 \rho+0.44147$. 


\subsubsection{Permeability}

Shale permeability refers to the ability of the rock to allow gas to pass, given a particular pressure difference. When it comes to the physical properties of a shale gas reservoir, shale permeability plays as an essential part in the evaluation of the horizontal well section. The value for this can be obtained from well completion data.

\subsubsection{Porosity}

Porosity relates to the main shale gas reservoir space. It is an important indicator because its size often controls the free shale gas content, which has a positive correlation with the total shale gas content. The larger the porosity, the greater the gas storage capacity. The value for this can again be obtained from well completion data.

\subsubsection{Gas Content}

Gas content is a key indicator for evaluating whether the shale gas is abundant enough for economic exploitation or at least possessed of sufficient potential. The gas content of shale gas refers to both the free gas and adsorbed gas. The higher the gas content, the better the gas content of the reservoir. Its value can be calculated by combining the TOC content with an existing empirical equation [14].

\subsection{Characteristics of the Sweet Spot Indicator System}

The evaluation indicator system is divided into three evaluation levels: good; medium; and poor. "Good" implies a "high quality" horizontal well section and is indicative of the prospective site being a priority area for fracturing. "Medium" can defined as a "normal" horizontal well section, which has less productive capability than "Good", but that is still worth fracturing. "Poor" indicates that an area should be abandoned, even if it is considered to have development potential. By way of an example, according to previous studies $[9,15,16]$, the shale gas horizontal well sections in the Fuling area can be evaluated as indicated in Tab. 1.

Table 1: Shale gas horizontal well section evaluation system

\begin{tabular}{llllllll}
\hline $\begin{array}{l}\text { Evaluation } \\
\text { indicator }\end{array}$ & $\begin{array}{l}\text { Brittleness } \\
\text { indicator }(\%)\end{array}$ & $\begin{array}{l}\text { Toughness } \\
\text { indicator }-\end{array}$ & $\begin{array}{l}\text { Fracturing } \\
\text { indicator }-\end{array}$ & $\begin{array}{l}\text { TOC }(\%) \\
\text { Permeability Porosity } \\
\left(10^{-3} \mathrm{mD}\right)\end{array}$ & $\begin{array}{l}\text { Gas content } \\
\left(\mathrm{m}^{3} \cdot \mathrm{t}^{-1}\right)\end{array}$ \\
\hline Good & $\geq 45$ & $\leq 1.9$ & $\geq 0.4$ & $\geq 3$ & $\geq 100$ & $\geq 5$ & $\geq 4$ \\
Medium & $40-45$ & $1.9-3.5$ & $0.35-0.4$ & $2-3$ & $10-100$ & $2-5$ & $2-4$ \\
Poor & $<40$ & $>3.5$ & $<0.35$ & $<2$ & $<10$ & $<2$ & $<2$ \\
\hline
\end{tabular}

\section{Evaluation Model for Horizontal Well Sections}

Establishing an evaluation model for horizontal well sections can be divided into three steps: 1) calculating the value of each evaluation indicator and normalizing it; 2) calculating the weight to be given to each evaluation indicator; 3) comparison of the corresponding indicator weights with the standard indicator weights. The various values are multiplied and summed (see Eq. (20) for further detail). The process for evaluating the indicator values was set out in Section 2.1. There are three methods for determining the weights: subjective methods; objective methods; and a combination of subjective and objective weighting methods. Subjective weighting methods include expert surveys, analytical hierarchy processes (AHP), the use of binomial coefficients, chain ratio scoring, least square methods, etc. Objective methods include principal component analysis, entropy calculations, dispersion, mean square deviation, and multi-objective planning. Combined methods necessitate using a certain proportion of subjective and objective weighting methods. To establish the evaluation model for 
horizontal well sections, the weights need to take into account not only the opinions of field experts, but also the objective regularity of the indicators themselves. So, a combined weighting method is more in line with actual practice. However, it is difficult to determine the right proportion of subjective and objective weights in combined weighting methods, because decisions of relative importance are themselves subjective. So, to minimize the deviation between subjective and objective weights as much as possible, this paper advocates using a combined weighting method based on game theory. For the subjective weights, AHP is adopted. For the objective weights, entropy is used. The specific steps followed are given below.

\subsection{Determining the Subjective Weight}

The specific steps to determine the subjective weight by using AHP [17-19] and consulting experts are as follows:

\subsubsection{Build a Hierarchical Model, as Shown in Fig. 1}

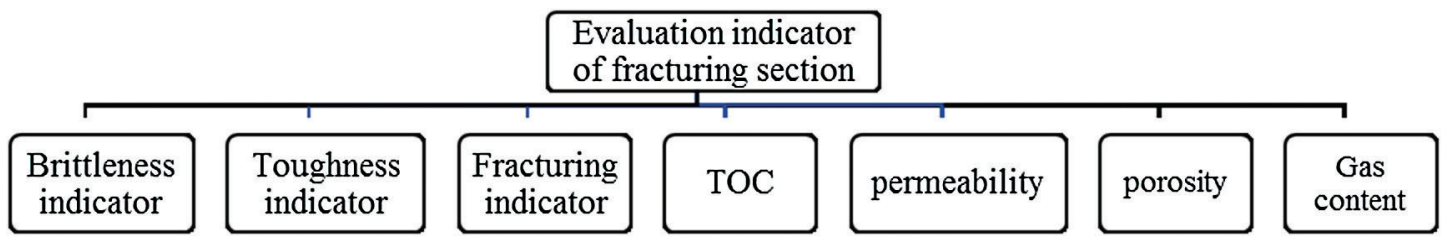

Figure 1: Hierarchical model

\subsubsection{Construct a Judgment Matrix}

A judgment matrix is constructed to compare pairs of indicator values so as to minimize the difficulty of comparing different factors with different properties. This produces a more accurate outcome. In general, the constraints given in Tab. 2 need to be met [20].

Table 2: Judgment conditions

\begin{tabular}{ll}
\hline Scale & Meaning \\
\hline 1 & The two indicators are equally important \\
3 & Compared with two indicators, one is slightly important than the other \\
5 & Compared with two indicators, one is Obviously important than the other \\
7 & Compared with two indicators, one is Strongly important than the other \\
9 & Compared with two indicators, one is Extremely important than the other \\
$2,4,6,8$ & The median value of the above two adjacent judgments \\
Reciprocal & $\begin{array}{l}\text { If the judgment of factor } i \text { compared with } j \text { is } a_{i j} \text {, then the judgment of factor } j \text { compared with } \\
i \text { is } a_{j i}\end{array}$ \\
\hline
\end{tabular}

\subsubsection{Conduct a Consistency Test}

(1) The consistency indicator, $C I$, can be calculated as follows:

$C I=\frac{\lambda_{\max }-n}{n-1}$.

$n$ is the order of the judgment matrix; and $\lambda_{\max }$ is the judgment matrix's maximum eigenvalue. 
(2) To calculate the consistency indicator, $R I$, a random method is adopted that involves constructing 500 sample matrixes so as to randomly select numbers and reciprocal numbers from 1 to 9 that can be used to construct a positive reciprocal matrix. The average maximum eigenvalue can be obtained as follows:

$R I=\frac{\lambda_{\max }-n}{n-1}$.

For the $R I$ value, $n=1-9$ (see Tab. 3).

Table 3: Corresponding $R I$ values for a matrix of order $n$

\begin{tabular}{llllllllllll}
\hline$n$ & 1 & 2 & 3 & 4 & 5 & 6 & 7 & 8 & 9 & 10 & 11 \\
$R I$ & 0 & 0 & 0.58 & 0.90 & 1.12 & 1.24 & 1.32 & 1.41 & 1.45 & 1.49 & 1.52 \\
\hline
\end{tabular}

(3) With regard to the consistency ratio, $C R$, when $C R<0.10$, the consistency of the judgment matrix is acceptable. Otherwise, the judgment matrix needs to be modified.

$C R=\frac{C I}{R I}$.

By using the above methods, (1)-(3), and Eqs. (7)-(9), the weight of the AHP can be calculated. The results are shown in Tab. 4, with the relative weights from the brittleness indicator to the gas content being as follows:

$w_{1}=(0.09,0.05,0.28,0.10,0.04,0.11,0.32)$

Table 4: Indicator matrix and weight vector

\begin{tabular}{|c|c|c|c|c|c|c|c|c|}
\hline Indicator & $\begin{array}{l}\text { Brittleness } \\
\text { indicator }\end{array}$ & $\begin{array}{l}\text { Toughness } \\
\text { indicator }\end{array}$ & $\begin{array}{l}\text { Fracturing } \\
\text { indicator }\end{array}$ & TOC & Permeability & Porosity & $\begin{array}{l}\text { Gas } \\
\text { content }\end{array}$ & Weight \\
\hline Brittleness indicator & 1 & 1 & $1 / 3$ & 1 & 3 & 1 & $1 / 5$ & 0.0878 \\
\hline Toughness indicator & 1 & 1 & $1 / 5$ & $1 / 3$ & 1 & $1 / 3$ & $1 / 5$ & 0.0510 \\
\hline Fracturing indicator & 3 & 5 & 1 & 3 & 5 & 3 & 1 & 0.2802 \\
\hline TOC & 1 & 3 & $1 / 3$ & 1 & 3 & 1 & $1 / 5$ & 0.1027 \\
\hline Permeability & $1 / 3$ & 1 & $1 / 5$ & $1 / 3$ & 1 & $1 / 3$ & $1 / 5$ & 0.0436 \\
\hline Porosity & 1 & 3 & $1 / 3$ & 1 & 3 & 1 & $1 / 3$ & 0.1105 \\
\hline Gas content & 5 & 5 & 1 & 5 & 5 & 3 & 1 & 0.3243 \\
\hline Consistency test & \multicolumn{8}{|c|}{ Maximum eigenvalue $=7.2267$ Consistency ratio $=0.0277 \mathrm{CI}=0.03778<0.1$} \\
\hline
\end{tabular}

\subsection{Determining the Objective Weight}

Entropy [21-24] was first put forward as an evaluation method by the German scholar, Klaus, in 1850. It can objectively reflect the impact of various factors used for evaluation on an evaluated object. The entropy is evaluated by selecting $m$ objects and $N$ indexes. By calculating the evaluation indexes of the different evaluation objects, we can compare their importance. The weight of the index is determined by the relative change in the degree of the index. When using entropy, the weight is positively related to the relative degree of change in the index. The entropy data matrix, $\mathrm{A}$, is as follows: 
$A=\left[\begin{array}{cccc}X_{11} & X_{12} & \ldots & X_{1 \mathrm{n}} \\ X_{21} & X_{22} & \ldots & X_{2 n} \\ \vdots & \vdots & \ddots & \vdots \\ X_{m 1} & X_{m 2} & \cdots & X_{m n}\end{array}\right]$

In Eq. (10), $X_{i j}$ is the value of the index, $j$, of an object, $i$, in the selected evaluation data. The specific steps for calculating entropy are as follow:

3.2.1 Calculate the Proportion of Scheme i Covered by Index $j$ in the Evaluation Data

$p_{i j}=\frac{\chi_{i j}}{\sum_{i=1}^{m} \chi_{i j}}$.

3.2.2 Calculate the Total Contribution of Index $j$ to the Attribute X in All Schemes

$E_{j}=-K \sum_{i=1}^{m} P_{i j} \ln \left(P_{i j}\right)$.

$K=\frac{1}{\ln (m)}$

\subsubsection{Calculate the Weight}

It can be seen from Eq. (12) that, when the relative contribution of an attribute to each scheme is more or less the same, $E_{i}$ will tend towards 1 . As the contribution becomes equal, the effect exerted by the attribute upon making a decision can be set aside. Thus, the weight of the attribute will be treated as zero. This also implies that the difference in size of an attribute's values across all schemes will determine its weight coefficient. The degree of the weight coefficient, $d_{j}$, can be therefore defined as relating to the consistency of the contribution made by the $j$-th attribute to each scheme [19]:

$d_{j}=1-E_{j}$.

The weight, $W_{J}$, of each attribute can then be described as:

$W_{J}=\frac{d_{j}}{\sum_{j=1}^{m} d_{j}}$.

On the basis of this, the logging and completion data for four wells, A1, A2, A3 and A4, in the Fuling area, were used to calculate the average value of the relevant parameters according to Eqs. (1)-(6). The decision matrix was then established using Eq. (10), as shown in Tab. 5.

Then, the matrix, $p$, was then calculated by using Eq. (11), as shown in Tab. 6.

Finally, by applying Eqs. (12)-(15), the weight of each indicator was obtained in sequence, as follows:

$w_{2}=(0.12,0.08,0.22,0.11,0.27,0.15,0.06)$ 
Table 5: Decision matrix parameters

\begin{tabular}{llllllll}
\hline Indicator & $\begin{array}{l}\text { Brittleness } \\
\text { indicator }(\%)\end{array}$ & $\begin{array}{l}\text { Toughness } \\
\text { indicator - }\end{array}$ & $\begin{array}{l}\text { Fracturing } \\
\text { indicator }-\end{array}$ & $\begin{array}{l}\text { TOC } \\
(\%)\end{array}$ & $\begin{array}{l}\text { Permeability } \\
\left(10^{-3} \mathrm{mD}\right)\end{array}$ & $\begin{array}{l}\text { Porosity } \\
(\%)\end{array}$ & $\begin{array}{l}\text { Gas content } \\
\left(\mathrm{m}^{3} \cdot \mathrm{t}^{-1}\right)\end{array}$ \\
\hline A1 & 52 & 0.62 & 1.74 & 3.4 & 0.21 & 4.8 & 4.8 \\
A2 & 61 & 0.83 & 1.34 & 2.9 & 0.23 & 4.2 & 5.2 \\
A3 & 51 & 0.77 & 1.82 & 3.2 & 0.27 & 5.3 & 5.5 \\
A4 & 62 & 0.92 & 2.1 & 3.5 & 0.28 & 4.7 & 4.9 \\
\hline
\end{tabular}

Table 6: P Matrix parameter

\begin{tabular}{llllllll}
\hline Indicator & $\begin{array}{l}\text { Brittleness } \\
\text { indicator }\end{array}$ & $\begin{array}{l}\text { Toughness } \\
\text { indicator }\end{array}$ & $\begin{array}{l}\text { Fracturing } \\
\text { indicator }\end{array}$ & TOC & Permeability & Porosity & $\begin{array}{l}\text { Gas } \\
\text { content }\end{array}$ \\
\hline A1 & 0.23 & 0.20 & 0.25 & 0.26 & 0.21 & 0.25 & 0.24 \\
A2 & 0.27 & 0.26 & 0.19 & 0.22 & 0.23 & 0.22 & 0.25 \\
A3 & 0.23 & 0.25 & 0.26 & 0.25 & 0.27 & 0.28 & 0.27 \\
A4 & 0.27 & 0.29 & 0.30 & 0.27 & 0.28 & 0.25 & 0.24 \\
\hline
\end{tabular}

\subsection{Combined Weighting Method Based on Game Theory}

Game theory [25] analyzes the rational behavior and equilibrium decision of multiple decision-makers when they interact with each other. In game theory, it is assumed that every scheme is the result of rational decision-making, which is based upon a decision-maker's desire to maximize their own gains or minimize their own losses. This kind of competitive result is not controlled by one decision maker, but rather realized by all decision makers. In the decision-making process, when all parties of the game coordinate to find their maximum common interest, there will be a compromise. Establishing the Nash equilibrium involves finding the consistency and compromise between different weights, i.e., finding the minimum deviation between the combined weight and each individual weight. This enables the sum of the deviations to be minimized, so that the maximum common interest can be achieved. The steps required to arrive at a combined weighting based on game theory are as follows:

\subsubsection{Devising the Composite Weight Set}

For a basic set of weight vectors, $U \mid=\left\{u_{1}, u_{2}, \cdots, u_{n}\right\}, n$ vectors are arbitrarily linearly combined into a possible weight set:

$U=\sum_{k=1}^{n} \alpha_{k} u_{k}^{T}, \alpha_{k}>0$

where: $u-\mathrm{A}$ is the possible weight vector of the set of possible weight vectors; and $\alpha_{k}$ is the weight coefficient.

\subsubsection{Finding the Most Satisfactory Weight Vector}

Using game theory, we can find the most satisfactory vector, $u^{*}$, in the possible vector set. The basic idea is to find consistency or compromise between different weights. Finding the most satisfactory weight vector can be transformed into optimizing the linear combined weight coefficient, $\alpha_{k}$. The objective of this optimization is to minimize the difference between $u$ and each $u_{k}$. Namely: 
$\min \left\|\sum_{j=1}^{n} \alpha_{j} \times u_{j}^{T}-u_{i}^{T}\right\|_{2}(i=1,2, \cdots, n)$

According to the differential properties of the matrix, the first optimization condition in Eq. (17) can be obtained as follows:

$\sum_{j=1}^{n} \alpha_{j} \times u_{i} \times u_{j}^{T}=u_{i} \times u_{i}^{T}(i=1,2, \cdots, n)$.

Eq. (18) corresponds to the following linear equations:

$$
\left[\begin{array}{cccc}
u_{1} u_{1}^{T} & u_{1} u_{2}^{T} & \cdots & u_{1} u_{m}^{T} \\
u_{2} u_{1}^{T} & u_{2} u_{2}^{T} & \cdots & u_{2} u_{n}^{T} \\
\vdots & \vdots & \vdots & \vdots \\
u_{n} u_{1}^{T} & u_{n} u_{2}^{T} & \cdots & u_{n} u_{n}^{T}
\end{array}\right]\left[\begin{array}{c}
\alpha_{1} \\
\alpha_{1} \\
\vdots \\
\alpha_{n}
\end{array}\right]=\left[\begin{array}{c}
u_{1} u_{1}^{T} \\
u_{2} u_{2}^{T} \\
\vdots \\
u_{n} u_{n}^{T}
\end{array}\right] .
$$

After the solution for $a_{i}$ in Eq. (19) has been normalized and substituted into Eq. (16), the comprehensive weight vector, $u$, can be obtained.

By using the above method, the subjective and objective weights, $w_{1}$ and $w_{2}$, can be introduced into Eq. (18) to get the weight coefficients $\alpha=0.702$ and $\beta=0.478$, with their normalized values being $\alpha=0.0 .595$ and $\beta=0.405$. The combined weighting method based on game theory can then be used to obtain the comprehensive weight, $u$, from Eq. (16) for each indicator:

$u=(0.1022,0.0622,0.2557,0.1041,0.1332,0.1262,0.2146)$

Thus, the relative importance of the different sweet spot indicators in order is: the fracturing indicator; the gas content; the porosity; the TOC; the brittleness indicator; the permeability and the toughness indicator.

\subsection{Establishing the Model}

By using the combined weight, the weight of each indicator can be obtained so as to establish a model for calculating the section evaluation indicator $(\mathrm{FI})$ :

$F I=0.1022 B_{1}+0.0622 B_{2}+0.2557 B_{3}+0.1041 B_{4}+0.1332 B_{5}+0.1262 B_{6}+0.2146 B_{7}$.

The normalized brittleness indicator and the normalized toughness indicator are $B_{1}$ and $B_{2}$, respectively; $B_{3}$ represents the normalized fracturing indicator; while $B_{4}$ is the normalized TOC. $B_{5}$ is the normalized permeability and $B_{6}$ is the normalized porosity, with $B_{7}$ being the normalized gas content.

$B_{1} B_{3} B_{4} B_{5} B_{6} B_{7}$ are the 6 positive indicators, which can be standardized as follows:

$S=\frac{B-B_{\min }}{B_{\max }-B_{\min }}$.

$B_{2}$ is a negative indicator, which can be standardized as:

$S=\frac{B_{\max }-B}{B_{\max }-B_{\min }}$. 


\section{Model Verification and Application}

As mentioned above, Well A1 in the Fuling District of Chongqing was used as a test example by applying the evaluation model to its horizontal well section. The completion depth of Well A1 was 4015 $\mathrm{m}$ and the test section was $2540-3980 \mathrm{~m}$. The original data was gathered by carrying out conventional acoustic and element capture logging. The results from the logs were used to calculate the evaluation indicators for the relevant horizontal well sections by applying Eqs. (1)-(6). The results were then standardized by using Eqs. (21)-(22). On the basis of the evaluation system presented in Tab. 1, two sets of baseline data were obtained, i.e., the brittleness indicator, toughness indicator, fracturing ability indicator, organic carbon content, permeability, porosity and gas content. The values for these were: 45; $1.9 ; 0.4 ; 3100 ; 5 ; 4 \& 40 ; 3.5 ; 0.3 ; 2 ; 10 ; 2$; and 2 . These values were used as the baseline data for Eq. (20), enabling the two baseline values of 0.61 and 0.25 to be obtained. Finally, by combining the combined weight and standardized section evaluation indicators using Eq. (20), evaluation indicators for the Well A1 section were obtained, with their values ranging between 0.18 and 0.81 , with an average value of 0.48 .

The results of using the section evaluation model to evaluate the test section of Well A1 are shown in Fig. 2. It can be seen that there is a positive correlation between the evaluation indicators for the horizontal well section and the brittleness indicator, fracturing indicator, organic carbon content, permeability, porosity and gas content. In other words, the higher the evaluation indicator for the horizontal well section, the higher the brittleness indicator and fracturing indicator, the higher the content of brittle minerals, and the better the horizontal well section's fracturing ability. Alongside of this, the higher the content of organic carbon, the permeability, porosity and gas content, the better the physical properties of the target layer and the more valuable the development. However, there was a negative correlation between the evaluation indicators for the horizontal well section and the toughness indicator. So, the greater the toughness of the rock, the less likely it is to fracture effectively and the lower the evaluation indicator for the horizontal well section. It can also be seen from the figure that the two baselines divide the section evaluation indicator into three evaluation intervals: good, medium and poor. The areas with a value above 0.61 are the best positions for finding a sweet spot. The areas with a value below 0.25 are best abandoned. Apart from that, the horizontal section for Well A1 as a whole, largely has a value in the middle range, which means that is has good physical properties and is suitable for fracturing.

When compared with the FSI gas production profile of the well, there is a good positive correlation between the profile and the section evaluation indicators (see Fig. 3). All of the sections were seen to have production capability except the first, fourth, sixth and 15th sections, with the proportion of effective sections being $73 \%$. Were the section evaluation model to be adopted, the invalidity of the 4th and 6th sections would be evident. By focusing on an increased proportion of effective sections as a result of adopting this approach, costs could be reduced and the efficiency increased in the development of the gas field. 


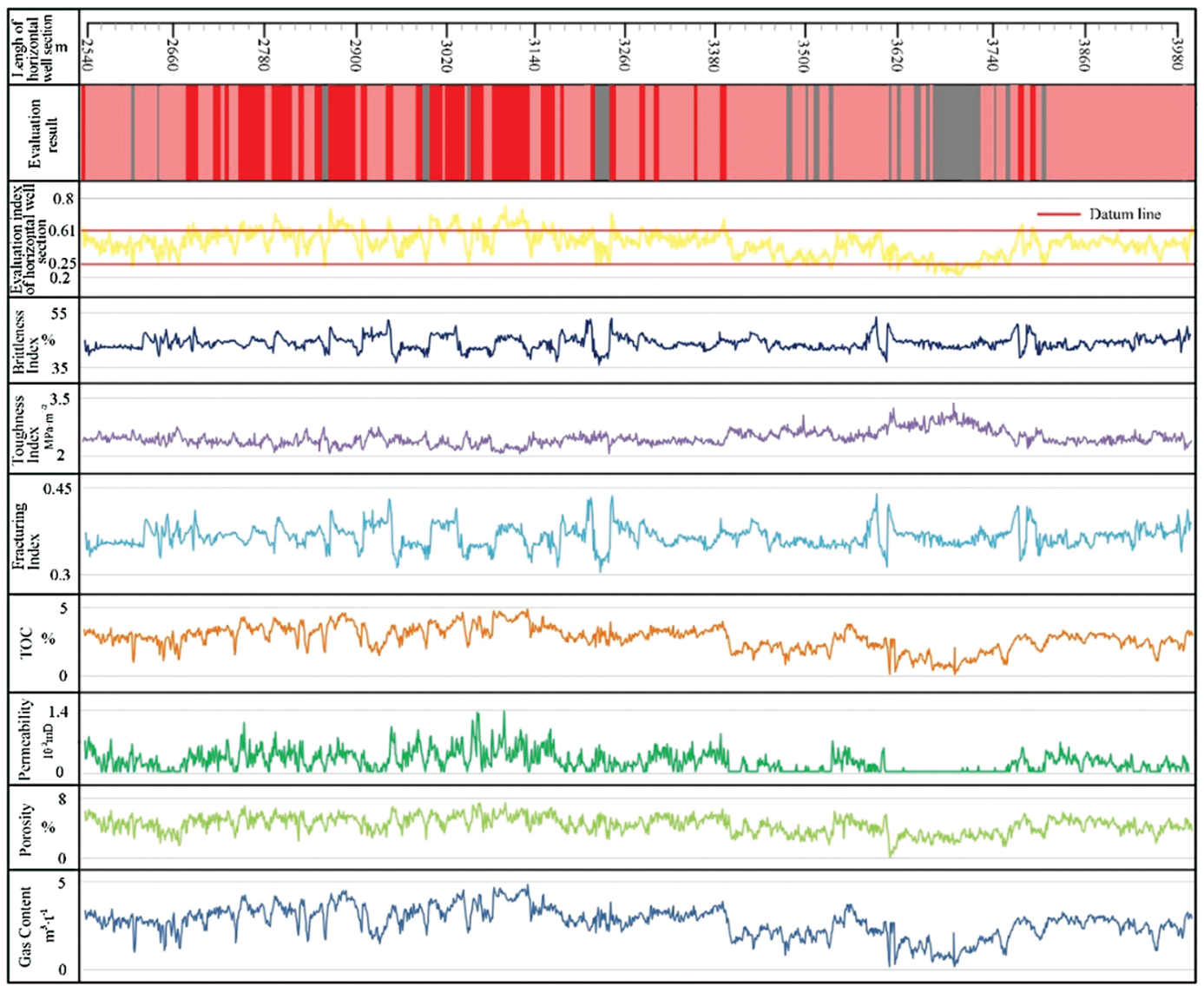

Figure 2: Evaluation diagram of horizontal well section

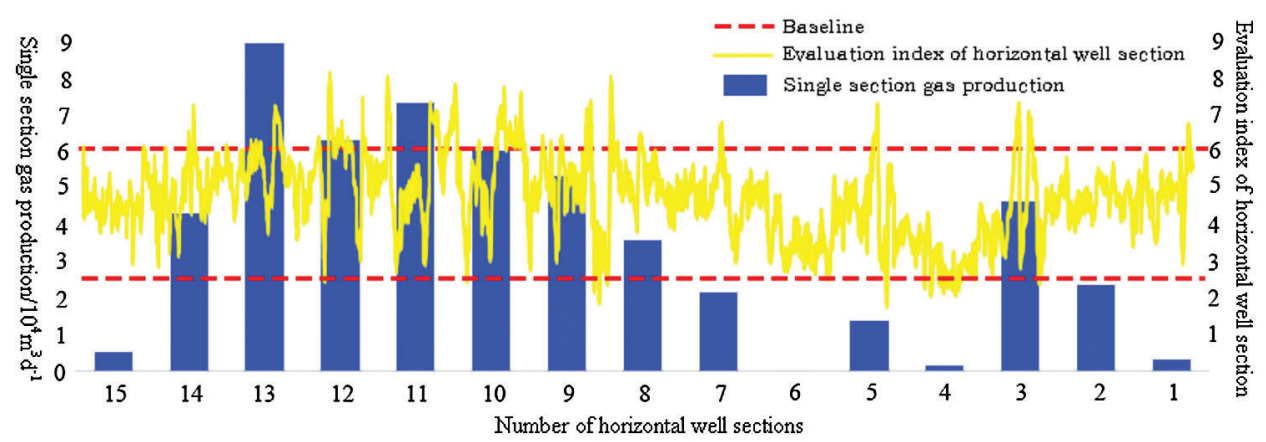

Figure 3: Well A1 section evaluation indicator and production period profile

\section{Conclusion}

(1) Existing indicators of engineering and geological sweet spots were derived from previous studies to form the basis of selecting appropriate sweet spot evaluation indicators for the development of a more effective evaluation model. These indicators were the rock's brittleness, toughness, fracturing capability, organic carbon content, porosity and permeability. Both subjective and objective factors were taken into account and an approach to combined weighting based on game 
theory was used to calculate the indicator weights. A horizontal well section evaluation model was then established by multiplying the standardized indicator values and combined weights.

(2) The approach was applied to Well A1 in the Fuling District of Chongqing and the relevant section evaluation indicators were obtained, which ranged from 0.18 to 0.81 with an average value of 0.48 . The horizontal section of Well A1 was found to have a medium rating, which implies that it has good physical properties and is suitable for fracturing. The obtained evaluation indicators were compared with the FSI gas production profile and they were found to have a good positive correlation. If the section evaluation model is adopted, exploitation of the poor quality 4th and 6th sections could be avoided and the proportion of effort devoted to effective sections increased, thereby reducing cost and increasing efficiency in the development of the gas field.

Acknowledgement: The authors would like to express their gratitude to EditSprings (https://www. editsprings.com/) for the expert linguistic services provided.

Funding Statement: This work was supported by the National Science and Technology Major Project during the 13th Five-Year Plan under grant (2016ZX05060-019) and the National Science and Technology Major Project during the 13th Five-Year Plan under grant (2016ZX05060004).

Conflicts of Interest: The authors declare that they have no conflicts of interest to report regarding the present study.

\section{References}

1. Zou, C. N., Dong, D. Z., Wang, J. Z., Li, J. Z., Li, X. J. et al. (2010). Geological characteristics, formation mechanism and resource potential of shale gas in China. Petroleum Exploration and Development, 37(6), 641653. DOI 10.1016/S1876-3804(11)60001-3.

2. Zeng, Q. C., Chen, S., He, P., Yang, Q., Guo, X. L. et al. (2018). Quantitative seismic prediction of shale gas sweet spots in Lower Si Chuan Longmaxi Formation, Weiyuan area, Sichuan Basin, SW China. Petroleum Exploration and Development, 45(3), 406-414.

3. Jiang, T. X., Bian, X. B. (2016). The novel technology of shale gas play evaluation-sweetness calculation method. Petroleum Drilling Techniques, 44(4), 1-6.

4. Liu, H. (2019). Prediction to shale gas sweet spot of Shanxi formation in Yan'an area (Master's Thesis). Journal of Xi'an University of Petroleum, China.

5. Liao, D. L., Lu, B. P. (2018). Evaluation method of engineering sweet spots of shale gas reservoir development: a case study from the Jiaoshiba Gas Field, Sichuan Basin. Petroleum Drilling Techniques, 32(2), 43-50.

6. Huang, J., Wu, L. Z., You, Y., Huang, X. K., Nie, B. et al. (2016). The evaluation and application of engineering sweet spots in a horizontal well in the fuling shale gas reservoir. Petroleum Drilling Techniques, 14(3), 16-20.

7. Wang, H. Q., Chen, J. B., Zhang, J., Xie, Q., Wei, B. et al. (2016). A New method of fracability evaluation of shale gas reservoir based on weight allocation. Petroleum Drilling Techniques, 44(3), 88-94.

8. Lai, F. Q., Luo, H., Qin, D. Y., Xia, W. X., Gong, D. J. (2018). Crushability evaluation of shale gas reservoir based on analytic hierarchy process. Special Oil \& Gas Reservoirs, 25(3), 154-159.

9. Zou, C. N., Dong, D. Z., Wang, Y. M., Li, X. J., Huang, J. L. et al. (2016). Shale gas in China: characteristics, challenges and prospects (II). Petroleum Exploration and Development, 43(2), 166-178. DOI 10.1016/S18763804(16)30022-2.

10. Jin, Y., Chen, M., Zhang, X. D. (2001). Determination of fracture toughness for deep well rock with geophysical logging data. Chinese Journal of Rock Mechanics and Engineering, 20(4), 454-456.

11. Lou, Y. S., Jin, Y. Q. (2006). Rock mechanics and petroleum engineering (monograph). China Petroleum Industry Press, pp. 95-103.

12. Yuan, J. L., Deng, J. G., Zhang, D., Li, D. H., Yan, W. et al. (2013). Fracability evaluation of shale gas reservoirs. Acta Petrolei Sinica, 34(3), 523-527. 
13. Shen, C., Ren, L., Zhao, J. Z., Tan, X. C., Wu, L. Z. (2017). A comprehensive evaluation indicator for shale reservoirs and its application: a case study of the Ordovician Wufeng Formation to Silurian Longmaxi Formation in southeastern margin of Sichuan Basin, SW China. Petroleum Exploration and Development, 44 (4), 649-658. DOI 10.1016/S1876-3804(17)30078-2.

14. Shi, W. R., Zhang, C. M., Zhang, Z. S., Xiao, S. K., Shi, Y. H. et al. (2015). Log evaluation of gas content from jiaoshiba shale gas reservoir in fuling gas field. Well Logging Technology, 39(3), 357-362.

15. Regulation of Shale Gas Resources/Reserves Estimation (2014). DZ/T 0254 standard. China Standardization Management Committee.

16. Li, W. G., Yang, S. L. (2011). An optimal system and evaluation methods of ranking shale gas development prospective zones. Natural Gas Industry, 31(4), 59-61.

17. Shaharuzaman., M. A., Sapuan., S. M., Mansor., M. R., Zuhri, M. Y. M. (2019). Decision support strategy in selecting natural fiber materials for automotive side-door impact beam. Journal of Renewable Materials, 7(10), 997-1010. DOI 10.32604/jrm.2019.07529.

18. Yusof, N. S. B., Sapuan, S. M., Sultan, M. T. H., Jawaid, M. (2020). Manufacturing process selection of "green” oil palm natural fiber reinforced polyurethane composites using hybrid tea criteria requirement and AHP method for automotive crash box. Journal of Renewable Materials, 8(6), 647-660. DOI 10.32604/jrm.2020.08309.

19. Lin, Z. C., Cheng, C. H. (2010). Creative design of multi-layer web frame structure using modified AHP and modified TRIZ clustering method. Computer Modeling in Engineering \& Sciences, 68(1), $25-54$.

20. Song, F. K. (2019). Research on Performance evaluation of the government decentralized purchasing (Master's Thesis). Southeast University, China.

21. Fan, Y., Liu, G. P. (2011). The circular economy development and characteristics of a comprehensive evaluation based on entropy method in gansu province. Mathematics in Practice and Theory, 41(16), 38-43.

22. Morsli, S., Sabeur, A., El Ganaoui, M. (2015). Numerical simulation of entropy generation in hydrogen-air burner. Fluid Dynamics \& Materials Processing, 11(4), 342-353.

23. Maougal, A., Bessaïh, R. (2013). Heat transfer and entropy analysis for mixed convection in a discretely heated porous square cavity. Fluid Dynamics \& Materials Processing, 9(1), 35-59.

24. Huo, H. M., Xu, W., Li, A. G. (2019). Comparison and analysis of envelope structure retrofitting schemes for rural residential building in northern china based on entropy method. Building Science, 35(8), 57-64.

25. Cui, C. S., Wang, Y., Li, Q. (2019). Performance evaluation of logistics service based on game theory indicator and comprehensive empowerment. Mathematics in Practice and Theory, 49(2), 110-118. 